\title{
Editorial
}

\section{Covid-19: A Global Challenge - Misinformation, Misconceptions And Myths}

\author{
Uchechukwu Levi Osuagwu (PhD, MSc, OD), \\ Diabetes, Obesity and Metabolism Translational Research Unit (DOMTRU), \\ School of Medicine, Western Sydney University, \\ Campbelltown New South Wales 2560 Australia.
}

\begin{abstract}
$\mathrm{T}$ he first quarter of the 21 st century will go down in history as one in which a global pandemic brought everything to a sudden halt. This pandemic, which started in Wuhan, China in December of 2019, was caused by the coronavirus (COVID-19), and spread like wild fire with over 100 million people infected worldwide. Nigeria was the first country in Sub-Saharan Africa to record its first case of COVID-19 on the 27 th of February 2020. It has since spread to many of the countries in this region who reacted by placing travel restrictions and lockdowns within their territories.
\end{abstract}

The lack of reliable information about the disease gave room for many theorists to propound their views and freely circulated them on social media and other internet channels. The medical world struggled to keep up with the misinformation, misconceptions and myths that had flooded the media space which in many cases were propagated by well-known political and religious personalities. This resulted in fear and panic particularly among the poor nations of the world.

This observation prompted a group of African scholars known as the AFRICAN TRANSLATION RESEARCH GROUP (ATReG), from different parts of the world to look into these misconceptions being spread about COVID-19 and how it affects the disease control and people compliance with the precautionary measures that were being advocated by health authorities.

With an online survey, ATReG investigated certain misinformation and myths that were prevalent at the period (April - May 2020) when most sub-Saharan countries were under mandatory lockdown and movement restrictions. The survey had over 1,000 participants and they responded to questions on the following misinformation about the disease:

1. COVID-19 is designed to reduce the world population

2. Your ability to hold your breath for 10 seconds means you do not have COVID-19

3. drinking hot water flushes the virus

4. the virus had little effect on blacks than whites and

5. $5 \mathrm{G}$ network was the cause of COVID-19

The findings of these studies, which were published in various international journals, indicated that one in every five people that responded believed either that COVID-19 was designed to reduce world population, or thought their ability to hold their breath for 10 seconds meant that they do not have COVID-19. For every four individuals, there was one person who believed that drinking hot water flushes down the virus, with another 14\% who thought that COVID-19 had little effect on Blacks than with Whites.

The significant number of Africans who believed these misconceptions were mostly older adults, women, those who had no job at the time of the study, people residing in East African countries like Kenya and Tanzania and those who were educated. According to the researchers,. Those who believe in the misinformation felt that their risk of catching the virus was low and were less likely to comply with the 
precautionary public health measures such as hand washing, wearing facemask and avoiding events with large gatherings.

Regarding the myths surrounding the $5 \mathrm{G}$ network and the coronavirus disease, a little over $7 \%$ of the respondents believed that the $5 \mathrm{G}$ network was behind the pandemic and these were mostly those living in Central Africa, young people, women, people with no jobs and those who thought that the pandemic would not continue in their countries. The papers with these results and their detailed information can be found on the following links: https://www.liebertpub.com/doi/10.1089/HS.2020.0202 and https://doi. org/10.29392/001c.17606.

The researchers concluded that this false information have far reaching implications and influence on individual actions. It also portends danger for future interventions such as vaccines and drug treatments, which are already being rolled out and highlight the need for health messages aimed at dispelling misinformation, misconceptions and myths. These messages should be tailor made to target specific subgroups as identified in these studies.

\section{Uchechukwu Levi Osuagwu (PhD, MSc, OD),}

Diabetes, Obesity and Metabolism Translational Research Unit (DOMTRU),

School of Medicine, Western Sydney University, Campbelltown New South Wales 2560 Australia.

Other team members include: Miner A. Chundung, Bernadine Ekpenyong, Piwuna Christopher Goson, Tanko Ishaya (Nigeria); Khathutshelo Percy Mashige (South Africa), Abu Kwasi Emmanuel (Ghana), Chikasirimobi G Timothy (Kenya), Godwin Ovenseri-Ogbomo (Saudi Arabia), Raymond, Langsi (Cameroon); Deborah Donald Charwe (Tanzania), Obinna Nweze(United Kingdom), Richard Oloruntoba and Kingsley Agho (Australia) 